Original paper

\title{
Task-based characterization of a deep learning image reconstruction and comparison with filtered back-projection and a partial model-based iterative reconstruction in abdominal CT: A phantom study
}

\author{
Damien Racine ${ }^{\mathrm{a}}$, Fabio Becce ${ }^{\mathrm{b}, 1}$, Anais Viry ${ }^{\mathrm{a}}$, Pascal Monnin ${ }^{\mathrm{a}}$, Brian Thomsen ${ }^{\mathrm{c}}$, \\ Francis R. Verdun ${ }^{\mathrm{a}}$, David C. Rotzinger ${ }^{\mathrm{b}, *, 1}$ \\ ${ }^{a}$ Institute of Radiation Physics (IRA), Lausanne University Hospital (CHUV) and University of Lausanne (UNIL), Rue du Grand-Pré 1, 1007 Lausanne, Switzerland \\ ${ }^{\mathrm{b}}$ Department of Diagnostic and Interventional Radiology, Lausanne University Hospital (CHUV) and University of Lausanne (UNIL), Rue du Bugnon 46, 1011 Lausanne, \\ Switzerland \\ ${ }^{\mathrm{c}}$ GE Healthcare, Milwaukee, WI, USA
}

\section{A R T I C L E I N F O}

\section{Keywords:}

Computed tomography

Deep learning

Image quality

Iterative reconstruction

Model observer

Radiation dose

\begin{abstract}
A B S T R A C T
Purpose: We aimed to thoroughly characterize image quality of a novel deep learning image reconstruction (DLIR), and investigate its potential for dose reduction in abdominal CT in comparison with filtered back-projection (FBP) and a partial model-based iterative reconstruction (ASiR-V).

Methods: We scanned a phantom at three dose levels: regular (7 mGy), low (3 mGy) and ultra-low (1 mGy). Images were reconstructed using DLIR (low, medium and high levels) and ASiR-V ( $0 \%=$ FBP, $50 \%$ and $100 \%$ ). Noise and contrast-dependent spatial resolution were characterized by computing noise power spectra and target transfer functions, respectively. Detectability indexes of simulated acute appendicitis or colonic diverticulitis (low contrast), and calcium-containing urinary stones (high contrast) $(|\Delta \mathrm{HU}|=50$ and 500 , respectively) were calculated using the nonprewhitening with eye filter model observer.

Results: At all dose levels, increasing DLIR and ASiR-V levels both markedly decreased noise magnitude compared with FBP, with DLIR low and medium maintaining noise texture overall. For both low- and high-contrast spatial resolution, DLIR not only maintained, but even slightly enhanced spatial resolution in comparison with FBP across all dose levels. Conversely, increasing ASiR-V impaired low-contrast spatial resolution compared with FBP. Overall, DLIR outperformed ASiR-V in all simulated clinical scenarios. For both low- and high-contrast diagnostic tasks, increasing DLIR substantially enhanced detectability at any dose and contrast levels for any simulated lesion size.

Conclusions: Unlike ASiR-V, DLIR substantially reduces noise while maintaining noise texture and slightly enhancing spatial resolution overall. DLIR outperforms ASiR-V by enabling higher detectability of both low- and high-contrast simulated abdominal lesions across all investigated dose levels.
\end{abstract}

\section{Introduction}

Computed tomography (CT) has evolved into an unrivaled diagnostic tool in many diseases and clinical scenarios, owing in particular to its wide availability, speed and diagnostic performance. In fact, almost a third of CT examinations involve the abdomen, and abdominal and pelvic CT has become a key player in the diagnosis and management of patients with acute abdominal pain [1]. However, its numerous benefits are overshadowed by constant radiation dose concerns - particularly in younger patients or those undergoing repeated CT scans
$[2,3]$ - considering that CT contributes more than half of the population's radiation exposure from medical imaging in Western countries [4].

Joint efforts to optimize CT protocols together with the successful implementation of iterative reconstruction (IR) techniques have enabled substantial dose reductions over the past decade in comparison with "analytical" filtered back-projection (FBP) algorithms [5,6], especially for CT examinations implying high-contrast diagnostic tasks [7-12]. However, the real impact of IR techniques on dose reduction in abdominal CT remains moderate at $\sim 20-30 \%$ [13-16], for the

\footnotetext{
* Corresponding author.

E-mail address: david.rotzinger@chuv.ch (D.C. Rotzinger).

${ }^{1}$ Fabio Becce and David C. Rotzinger contributed equally to this work.
} 


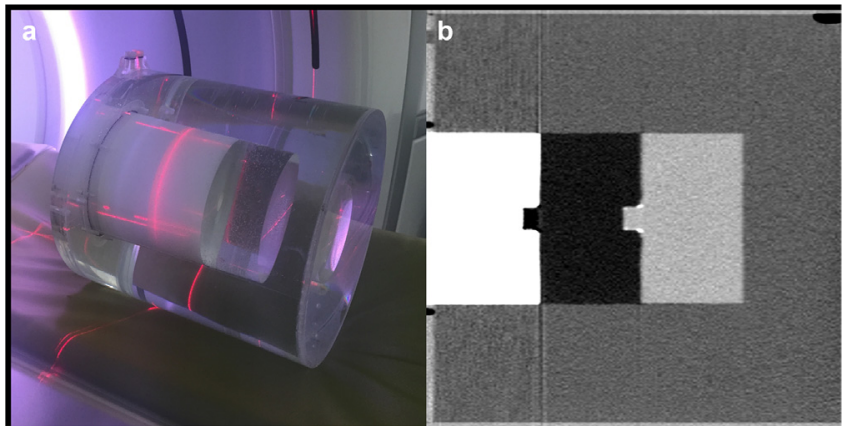

Fig. 1. (a) Photograph and (b) corresponding sagittal reformatted CT image of the custom 25-cm-diameter cylindrical phantom with its three 10-cm-diameter central cylindrical inserts made of PTFE, low-density PE and PMMA (from left to right), all surrounded by water. (b) Data were acquired at regular dose (7 mGy) and CT image reconstructed using DLIR high.

following reasons. First, the majority of CT-based diagnostic tasks in this region rely on low-contrast detectability, which limits tolerance for noisy CT images [13-16]. Secondly, statistical "hybrid" and partial model-based IR techniques not only reduce noise magnitude but also change noise texture, especially when used at high levels [17-19]. This results in CT images with an artificial "plastic" appearance, which are less well accepted by the radiology community and may affect diagnostic confidence [20,21]. Finally, although full model-based IR techniques can provide even higher noise reduction [22,23], their computing power requirements have thus far prevented widespread use and sustainability in increasingly busy clinical workflows.

Therefore, future efforts should continue to seek maximizing the use of information available in CT raw data in a clinically sustainable fashion, while keeping further dose reductions and radiological hazards in mind. This is one of the many problems that artificial intelligence could address in radiology, not only by enhancing CT image quality, but also by saving dose while speeding up image reconstruction time [24]. Although deep learning image reconstruction (DLIR) methods were originally described two decades ago [25], they could not be immediately translated into clinical practice because of the lack of training data and computing power. Both are now available and DLIR opens the door to a new era in medical image reconstruction, with the potential to address the major challenges and issues of both FBP and IR techniques. Specifically, while FBP fails to account for less-than-ideal CT system properties and may lead to higher radiation dose levels, model-based IR allows for substantial yet task-dependent dose reductions at the expense of impaired image texture and longer

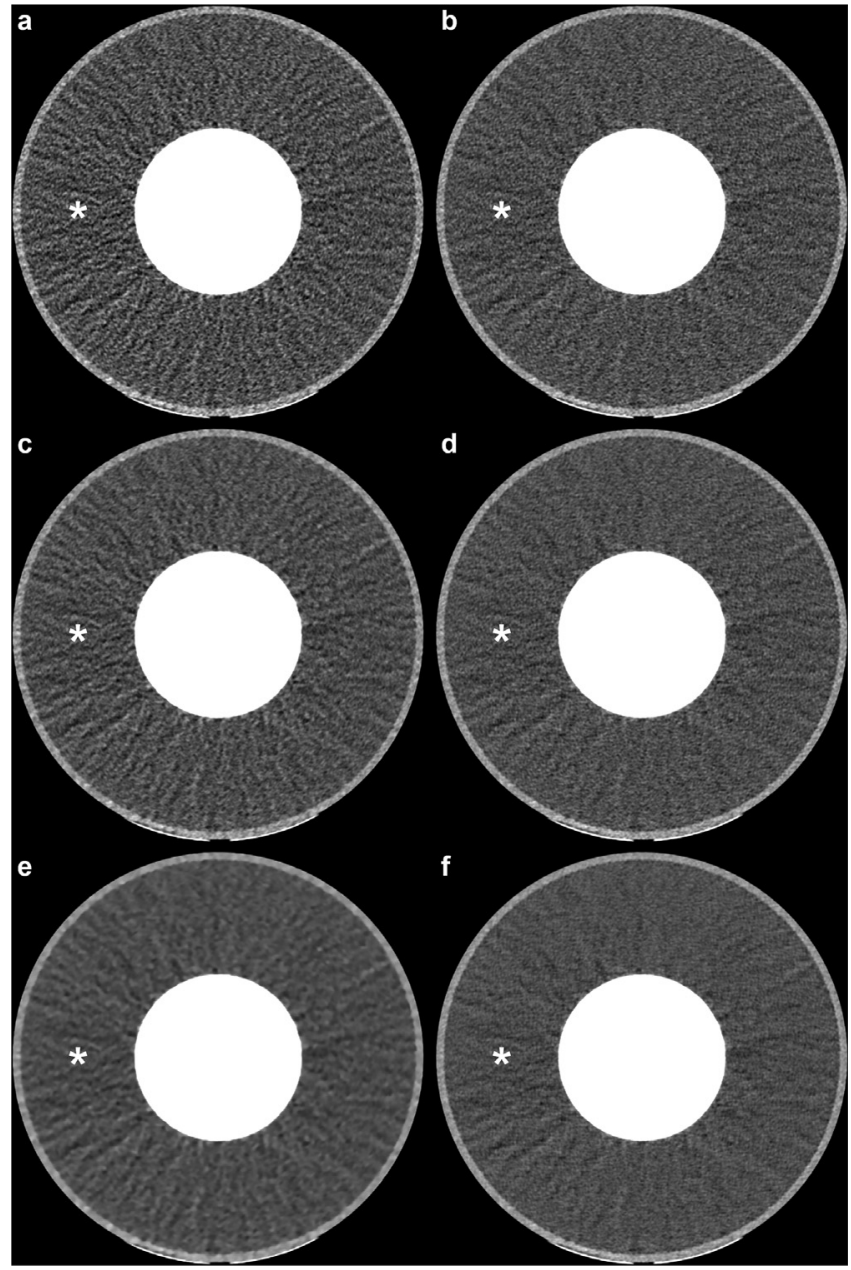

Fig. 2. Axial CT images of the custom 25-cm-diameter cylindrical phantom at mid-level of the 10-cm-diameter, 6-cm-height central cylindrical PTFE insert. Data were acquired at ultra-low dose (1 mGy) and images reconstructed using the following techniques: (a) ASiR-V 0\%, (c) ASiR-V 50\%, (e) ASiR-V 100\%, (b) DLIR low, (d) DLIR medium, and (f) DLIR high. Asterisks (*) indicate a homogeneous water region surrounding the PTFE insert. Note the impact of the various reconstruction algorithms and levels on noise magnitude and texture.

Table 1

Detailed settings for data acquisition and image reconstruction for the three investigated abdominal CT protocols (radiation dose levels).

\begin{tabular}{|c|c|c|c|}
\hline Radiation dose level & Regular & Low & Ultra-low \\
\hline CTDI $_{\text {vol }}$ (mGy) & 7 & 3 & 1 \\
\hline \multicolumn{4}{|l|}{ Data acquisition } \\
\hline Tube potential (kVp) & 100 & 100 & 100 \\
\hline Tube current (mA) & 360 & 155 & 50 \\
\hline Gantry revolution time (s) & 0.5 & 0.5 & 0.5 \\
\hline Beam collimation (mm) & $128 \times 0.625$ & $128 \times 0.625$ & $128 \times 0.625$ \\
\hline Pitch & 0.992 & 0.992 & 0.992 \\
\hline Scan field of view $(\mathrm{cm})$ & $50 \times 50$ & $50 \times 50$ & $50 \times 50$ \\
\hline \multicolumn{4}{|l|}{ Image reconstruction } \\
\hline Display field of view $(\mathrm{cm})$ & $32 \times 32$ & $32 \times 32$ & $32 \times 32$ \\
\hline Section thickness (mm) & 2.5 & 2.5 & 2.5 \\
\hline Section overlap (mm) & 1.25 & 1.25 & 1.25 \\
\hline Kernel & Standard & Standard & Standard \\
\hline \multirow{2}{*}{ Algorithm } & ASiR-V $0 \%, 50 \%$ and $100 \%$ & ASiR-V $0 \%, 50 \%$ and $100 \%$ & ASiR-V $0 \%, 50 \%$ and $100 \%$ \\
\hline & DLIR low, medium and high & DLIR low, medium and high & DLIR low, medium and high \\
\hline
\end{tabular}

ASiR-V = adaptive statistical iterative reconstruction, CTDI $_{\text {vol }}=$ volume CT dose index, DLIR = deep learning image reconstruction. In this study, ASiR-V 0\% was used as a surrogate for filtered back-projection. 

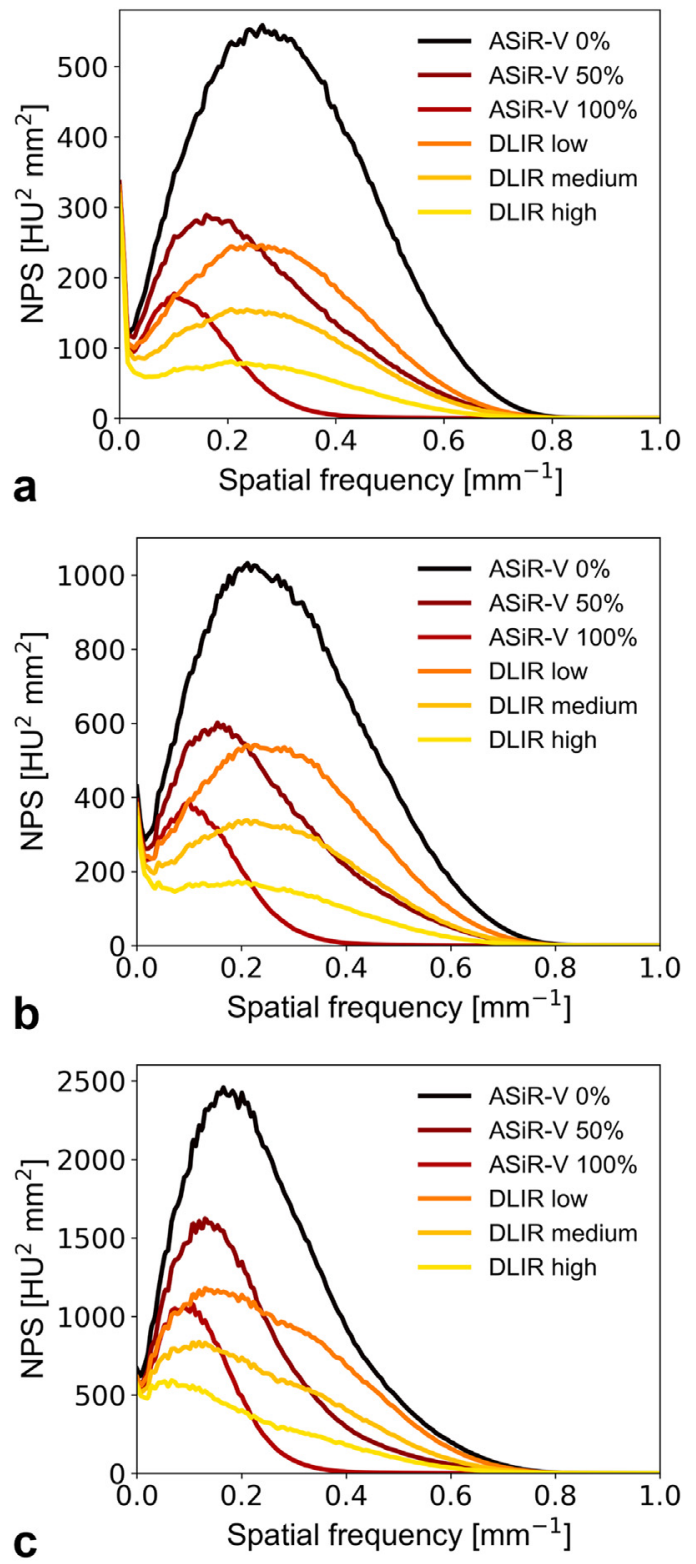

Fig. 3. NPS as a function of the image reconstruction technique and radiation dose level: (a) regular, (b) low and (c) ultra-low dose. At all three investigated dose levels, increasing DLIR and ASiR-V levels both markedly decreased noise magnitude (i.e. area under the curve) in comparison with FBP, with DLIR low and medium maintaining noise texture (i.e. peak frequency shift) overall. In this study, ASiR-V 0\% was used as a surrogate for FBP.

Table 2

Relative noise magnitude ratios (with corresponding percentage differences) for DLIR and ASiR-V levels in comparison with regular-dose FBP (ASiR-V 0\%) at the three investigated radiation dose levels.

\begin{tabular}{llll}
\hline $\begin{array}{l}\text { Radiation dose level } \\
\text { CTDI }_{\text {vol }}(\mathrm{mGy})\end{array}$ & $\begin{array}{l}\text { Regular } \\
7\end{array}$ & $\begin{array}{l}\text { Low } \\
3\end{array}$ & $\begin{array}{l}\text { Ultra-low } \\
1\end{array}$ \\
\hline Algorithm & & & \\
DLIR low & $0.68(-54 \%)$ & $0.99(-1 \%)$ & $1.43(+104 \%)$ \\
DLIR medium & $0.54(-71 \%)$ & $0.79(-38 \%)$ & $1.16(+34 \%)$ \\
DLIR high & $0.40(-84 \%)$ & $0.57(-68 \%)$ & $0.88(-23 \%)$ \\
ASiR-V 50\% & $0.67(-54 \%)$ & $0.92(-15 \%)$ & $1.36(+86 \%)$ \\
ASiR-V 100\% & $0.40(-84 \%)$ & $0.57(-68 \%)$ & $0.91(-17 \%)$ \\
\hline
\end{tabular}

ASiR-V = adaptive statistical iterative reconstruction, CTDI $_{\mathrm{vol}}=$ volume CT dose index, DLIR = deep learning image reconstruction, FBP = filtered backprojection. In this study, ASiR-V 0\% was used as a surrogate for FBP.
Table 3

NPS peak frequency shifts (percentage differences) for DLIR and ASiR-V levels in comparison with FBP (ASiR-V 0\%) at the three investigated radiation dose levels.

\begin{tabular}{|c|c|c|c|}
\hline Radiation dose level & Regular & Low & Ultra-low \\
\hline $\mathrm{CTDI}_{\mathrm{vol}}(\mathrm{mGy})$ & 7 & 3 & 1 \\
\hline \multicolumn{4}{|l|}{ Algorithm } \\
\hline DLIR low & $-6.0 \%$ & $-0.4 \%$ & $+5.8 \%$ \\
\hline DLIR medium & $-10.6 \%$ & $-5.5 \%$ & $-1.6 \%$ \\
\hline DLIR high & $-19.5 \%$ & $-15.7 \%$ & $-14.9 \%$ \\
\hline ASiR-V 50\% & $-18.0 \%$ & $-18.5 \%$ & $-18.8 \%$ \\
\hline ASiR-V 100\% & $-56.3 \%$ & $-54.7 \%$ & $-49.6 \%$ \\
\hline
\end{tabular}

ASiR-V = adaptive statistical iterative reconstruction, $\mathrm{CTDI}_{\mathrm{vol}}=$ volume CT dose index, DLIR = deep learning image reconstruction, $\mathrm{FBP}=$ filtered backprojection, NPS = noise power spectrum. In this study, ASiR-V $0 \%$ was used as a surrogate for FBP.

reconstruction time due to the mathematical complexity of the involved models. In contrast, owing to its computational efficiency and high potential to distinguish between signal and noise, DLIR is supposed to provide novel opportunities for additional dose savings at higher image reconstruction speed, while keeping CT image look natural and satisfactory for radiologists.

Here, we aimed to thoroughly characterize and quantify the impact of a novel DLIR method on CT image noise and contrast-dependent spatial resolution properties, and secondly assess its potential for dose reduction in abdominal CT in comparison with FBP and a partial modelbased IR technique. Specifically, the various CT protocols investigated in this phantom study using advanced image quality metrics were tailored to match a range of common and clinically relevant scenarios encountered by radiologists when using CT to evaluate patients with acute abdominal pain, including both low-contrast (suspected acute appendicitis or colonic diverticulitis) and high-contrast (suspected calcium-containing urinary stones) diagnostic tasks.

\section{Materials and methods}

\subsection{Experimental design and CT protocols}

We used a custom 25-cm-diameter cylindrical phantom with three 10-cm-diameter central cylindrical inserts to compute the target transfer function (TTF), an advanced image quality metric for contrastdependent spatial resolution. These central inserts were made of polytetrafluoroethylene (PTFE or Teflon ${ }^{\circledR}$, average CT number at $100 \mathrm{kVp} \approx 1250 \mathrm{HU}$ ), low-density polyethylene (PE, average CT number at $100 \mathrm{kVp} \approx-100 \mathrm{HU}$ ), and polymethylmethacrylate (PMMA or Plexiglas ${ }^{\circledast}$, average CT number at $100 \mathrm{kVp} \approx 120 \mathrm{HU}$ ), and were surrounded by water (Fig. 1) [26,27]. Only PE and PTFE inserts were analyzed in this study because these materials mimicked the absolute contrast differences $(|\Delta H U|)$ faced clinically by radiologists when evaluating abdominal and pelvic CT scans for suspected acute appendicitis or colonic diverticulitis (low-contrast diagnostic task) and calcium-containing urinary stones (high-contrast diagnostic task), respectively. One end of the phantom contained no central insert and was uniformly filled with water; this homogeneous water area was used to compute the noise power spectrum (NPS), a further advanced image quality metric for noise. Approval of the institutional ethics committee was not required for this phantom-only study.

The phantom was scanned on a 256-detector row CT system (Revolution $\mathrm{CT}^{\circledast}{ }^{\otimes}$, GE Healthcare) using standard institutional clinical CT protocols for various abdominal and pelvic diagnostic tasks, at two radiation dose levels: regular $(7 \mathrm{mGy}$, suspected acute appendicitis or colonic diverticulitis) and low (3 mGy, suspected urinary stones) dose. Although not used clinically yet, a third ultra-low ( $1 \mathrm{mGy}$ ) dose level was investigated to explore the potential for further dose reduction with 

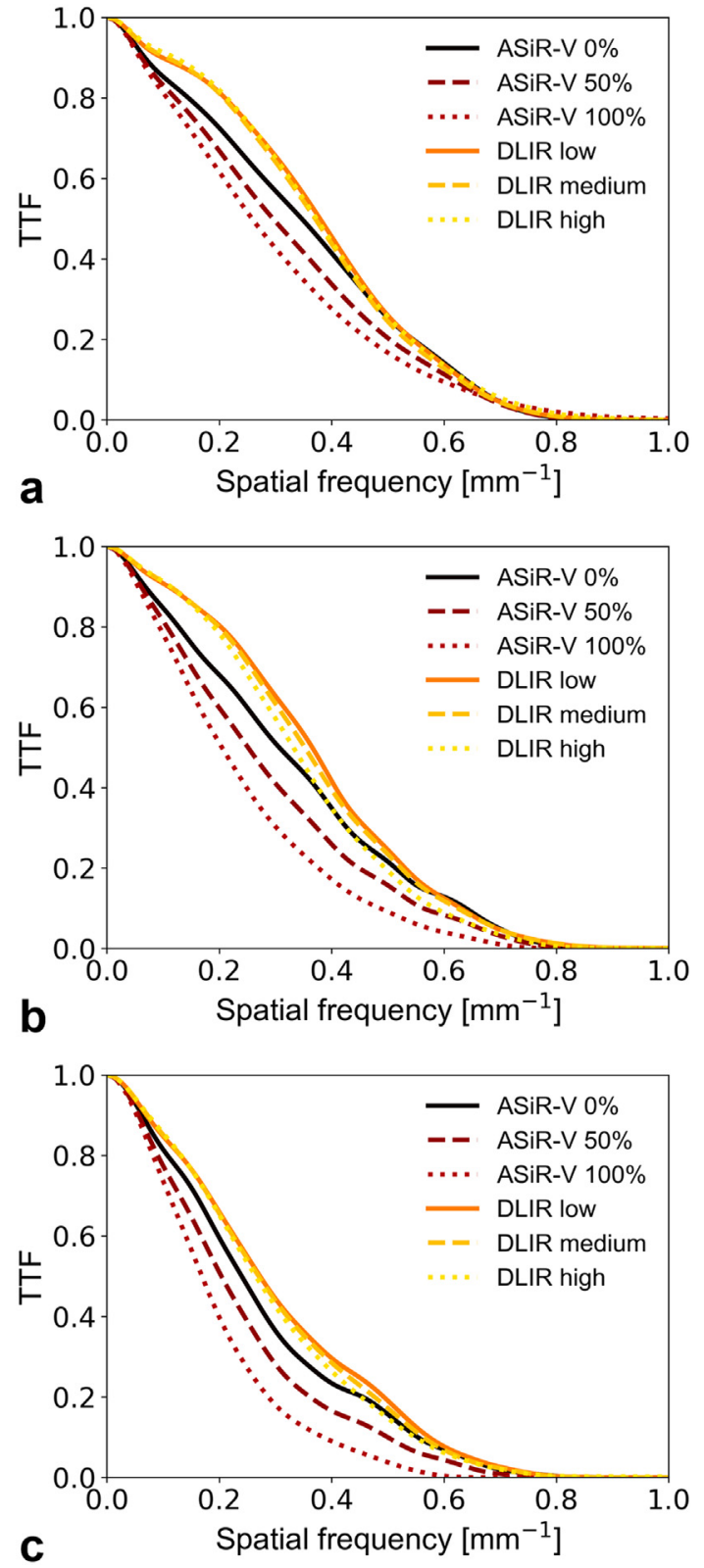

Fig. 4. Low-contrast TTF as a function of the image reconstruction technique and radiation dose level: (a) regular, (b) low and (c) ultra-low dose. DLIR not only maintained, but even enhanced low-contrast spatial resolution in comparison with FBP at all three investigated dose levels, particularly at low and ultra-low doses. Conversely, increasing ASiR-V levels impaired low-contrast spatial resolution compared with FBP. In this study, ASiR-V 0\% was used as a surrogate for FBP.

the novel DLIR algorithm. Volume CT dose indexes $\left(\mathrm{CTDI}_{\mathrm{vol}}\right)$ were calculated according to recommendations from the International Electrotechnical Commission (IEC 60601-2-44), and retrieved from radiation-dose structured reports. Detailed settings for CT data acquisition and image reconstruction are reported in Table 1. Phantom scanning was repeated five times consecutively without repositioning to provide a single dataset with a sufficient number of images for accurate computation of image quality metrics.

\section{Image reconstruction}

Images were reconstructed using both a DLIR (TrueFidelity ${ }^{\circledR}$, GE Healthcare) at low, medium and high levels, and a partial model-based
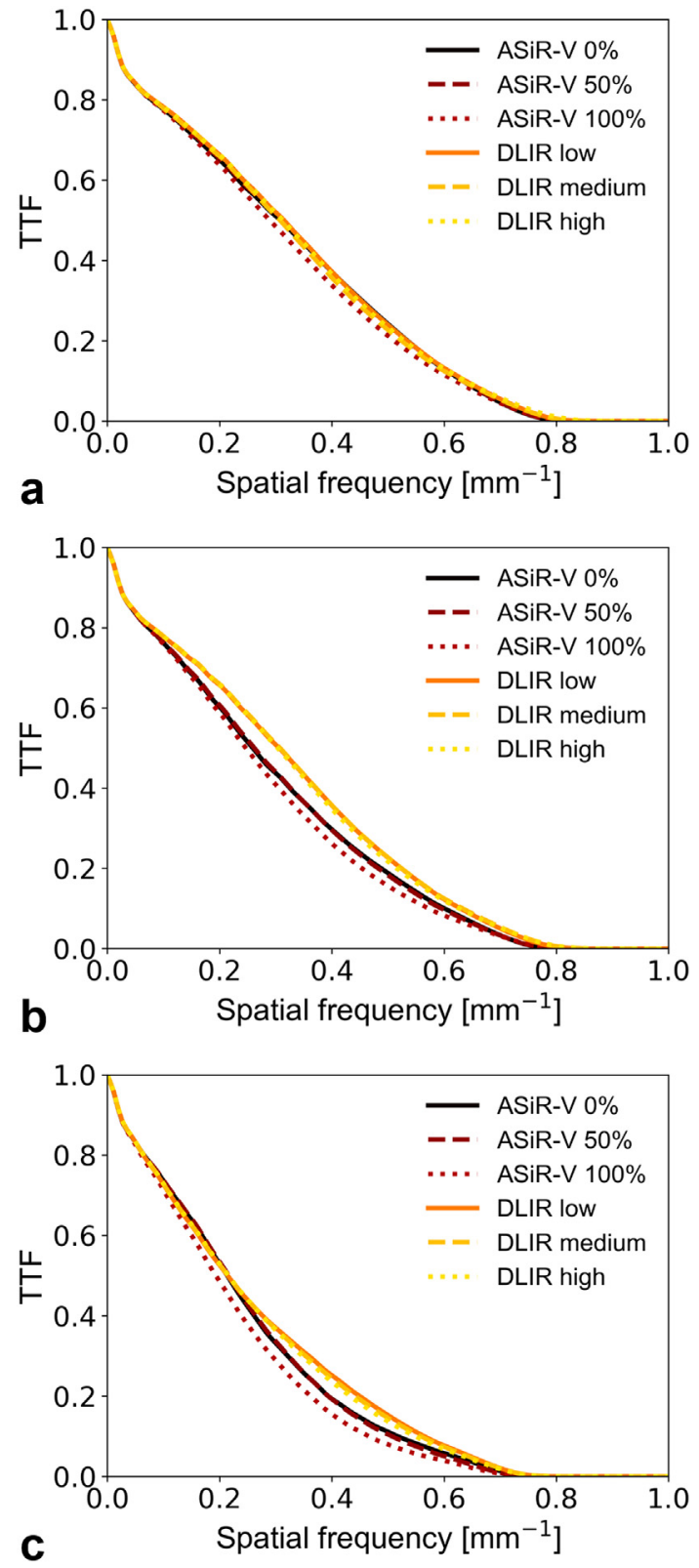

Fig. 5. High-contrast TTF as a function of the image reconstruction technique and radiation dose level: (a) regular, (b) low and (c) ultra-low dose. DLIR generally maintained high-contrast spatial resolution in comparison with FBP at regular dose, while slightly to moderately enhancing TTF at low and ultra-low doses. In contrast, increasing ASiR-V levels slightly decreased high-contrast spatial resolution at low and ultra-low doses. In this study, ASiR-V 0\% was used as a surrogate for FBP.

IR (adaptive statistical iterative reconstruction-V, ASiR-V ${ }^{\circledast}$, GE Healthcare) at $0 \%$ (used as a surrogate for FBP in this study), $50 \%$ and $100 \%$. This resulted in a total of 18 different CT datasets available for analysis: 3 dose levels (regular, low and ultra-low) $\times 2$ reconstruction techniques (DLIR and ASiR-V) $\times 3$ levels per reconstruction techniques (low, medium and high, or $0 \%, 50 \%$ and $100 \%$, respectively).

The ASiR-V image reconstruction technique is a mixture between a purely statistical IR algorithm (ASiR ${ }^{\circledR}, \mathrm{GE}$ Healthcare) [28] and a full model-based IR algorithm (Veo ${ }^{\circledR}$, GE Healthcare) [29]. Unlike the full model-based IR approach, ASiR-V decreases image reconstruction time by not modeling full system optics [30]. The ASiR-V algorithm can be prescribed at different levels from $0 \%$ to $100 \%$. ASiR-V $0 \%$ images are essentially FBP (no IR applied), while ASiR-V 100\% are maximum IR 
Table 4

Low-contrast TTF frequency shifts (percentage differences) for DLIR and ASiR-V levels in comparison with FBP (ASiR-V 0\%) at the three investigated radiation dose levels.

\begin{tabular}{|c|c|c|c|c|c|c|}
\hline \multirow{3}{*}{$\begin{array}{l}\text { Radiation dose } \\
\text { level } \\
\text { CTDI }_{\mathrm{vol}}(\mathrm{mGy})\end{array}$} & \multirow{2}{*}{\multicolumn{2}{|c|}{$\begin{array}{l}\text { Regular } \\
7\end{array}$}} & \multirow{2}{*}{\multicolumn{2}{|c|}{$\begin{array}{l}\text { Low } \\
3\end{array}$}} & \multirow{2}{*}{\multicolumn{2}{|c|}{$\begin{array}{l}\text { Ultra-low } \\
1\end{array}$}} \\
\hline & & & & & & \\
\hline & $\mathbf{T T F}_{50}$ & $\mathrm{TTF}_{10}$ & $\mathrm{TTF}_{50}$ & $\mathrm{TTF}_{10}$ & $\mathrm{TTF}_{50}$ & $\mathrm{TTF}_{10}$ \\
\hline \multicolumn{7}{|l|}{ Algorithm } \\
\hline DLIR low & $+9.6 \%$ & $-0.7 \%$ & $+18.7 \%$ & $-1.4 \%$ & $+13.9 \%$ & $+4.0 \%$ \\
\hline DLIR medium & $+7.0 \%$ & $-1.7 \%$ & $+14.4 \%$ & $-2.4 \%$ & $+13.0 \%$ & $+2.0 \%$ \\
\hline DLIR high & $+7.6 \%$ & $0 \%$ & $+7.9 \%$ & $-8.3 \%$ & $+11.1 \%$ & $-0.8 \%$ \\
\hline ASiR-V 50\% & $-14.6 \%$ & $-3.5 \%$ & $-18.7 \%$ & $-11.8 \%$ & $-14.8 \%$ & $-9.6 \%$ \\
\hline ASiR-V $100 \%$ & $-25.5 \%$ & $-7.6 \%$ & $-33.8 \%$ & $-23.9 \%$ & $-29.6 \%$ & $-30.4 \%$ \\
\hline
\end{tabular}

ASiR-V = adaptive statistical iterative reconstruction, $\mathrm{CTDI}_{\mathrm{vol}}=$ volume CT dose index, DLIR = deep learning image reconstruction, FBP = filtered backprojection, TTF $=$ target transfer function, $\mathrm{TTF}_{10}=$ target transfer function at $10 \%$ of its maximum value, $\mathrm{TTF}_{50}=$ target transfer function at $50 \%$ of its maximum value. In this study, ASiR-V 0\% was used as a surrogate for FBP.

Table 5

High-contrast TTF frequency shifts (percentage differences) for DLIR and ASiR$\mathrm{V}$ levels in comparison with FBP (ASiR-V 0\%) at the three investigated radiation dose levels.

\begin{tabular}{|c|c|c|c|c|c|c|}
\hline \multirow[t]{2}{*}{$\begin{array}{l}\text { Radiation dose level } \\
\mathrm{CTDI}_{\mathrm{vol}}(\mathrm{mG})\end{array}$} & \multicolumn{2}{|c|}{$\begin{array}{l}\text { Regular } \\
7\end{array}$} & \multicolumn{2}{|l|}{$\begin{array}{l}\text { Low } \\
3\end{array}$} & \multicolumn{2}{|c|}{$\begin{array}{l}\text { Ultra-low } \\
1\end{array}$} \\
\hline & $\mathrm{TTF}_{50}$ & $\mathrm{TTF}_{10}$ & $\mathrm{TTF}_{50}$ & $\mathrm{TTF}_{10}$ & $\mathrm{TTF}_{50}$ & $\mathrm{TTF}_{10}$ \\
\hline \multicolumn{7}{|l|}{ Algorithm } \\
\hline DLIR low & $+1.4 \%$ & $+0.7 \%$ & $+17.1 \%$ & $+4.8 \%$ & $+1.0 \%$ & $+9.4 \%$ \\
\hline DLIR medium & $-0.7 \%$ & $0 \%$ & $+18.8 \%$ & $+5.5 \%$ & $+1.0 \%$ & $+8.1 \%$ \\
\hline DLIR high & $+0.7 \%$ & $+0.7 \%$ & $+16.2 \%$ & $+4.4 \%$ & $+1.0 \%$ & $+6.0 \%$ \\
\hline ASiR-V 50\% & $0 \%$ & $0 \%$ & $+0.9 \%$ & $-1.1 \%$ & $+2.1 \%$ & $-2.1 \%$ \\
\hline ASiR-V 100\% & $0 \%$ & $-2.1 \%$ & $-5.1 \%$ & $-4.8 \%$ & $-9.4 \%$ & $-9.8 \%$ \\
\hline
\end{tabular}

ASiR-V = adaptive statistical iterative reconstruction, $\mathrm{CTDI}_{\mathrm{vol}}=$ volume CT dose index, DLIR = deep learning image reconstruction, FBP = filtered backprojection, $\mathrm{TTF}=$ target transfer function, $\mathrm{TTF}_{10}=$ target transfer function at $10 \%$ of its maximum value, $\mathrm{TTF}_{50}=$ target transfer function at $50 \%$ of its maximum value. In this study, ASiR-V $0 \%$ was used as a surrogate for FBP.

images. All other levels (\%) are a linear blend of these.

Deep learning reconstruction is a novel CT image reconstruction method that uses deep convolutional neural networks to reconstruct CT images. In this study, we evaluated a recently commercially-released DLIR algorithm from GE Healthcare (TrueFidelity ${ }^{\circledast}$ ) [31]. While in ASiR-V and other IR techniques engineers and scientists tune algorithms and manage parameters with the goal of modeling system behavior and ensuring iteration convergence, in the TrueFidelity algorithm, the models for the system behavior are implicitly generated through the training process, which allows for greater scale and incorporation of many more parameters into these models (i.e. millions of parameters). In any deep learning algorithm, the selection of the training dataset is critically important to determine the algorithm performance. With TrueFidelity, the training dataset included millions of both high- and low-dose CT samples from phantom studies performed in the manufacturer's laboratory and human studies with patients who underwent clinically indicated CT scans. The training dataset comprised different anatomical regions, CT protocols and patient morphologies. The highdose, low-noise dataset was reconstructed with FBP and used as a training target because under low-noise conditions, images produced by FBP are well accepted by the radiology community and exhibit a more natural look than IR images. In reconstructing images with TrueFidelity, three different levels can be selected to adjust the level of noise reduction: low, medium and high. This parameter may allow for setting image noise level to match preferences of radiologists with different experience levels and potentially to achieve dose reduction goals. These settings are designed to not impact noise texture, but noise magnitude.

\section{Image analysis}

\subsection{Noise power spectrum (NPS)}

Image noise was investigated in the insert-less, uniform water area of the phantom. Noise was quantified and characterized by computing NPS according to recommendations from the International Commission on Radiation Units and Measurements reports 54 and 87 [32,33]. A total of 165 axial CT sections were used for NPS computations. Square regions of interest (ROI) of $256 \times 256$ pixels were semi-automatedly extracted from the CT image center to obtain 2D NPS, which were then radially averaged to yield 1D NPS, as described in detail by Miéville et al. [34]. No detrending correction was applied to subtract large (beam hardening, scattering and bowtie) inhomogeneities prior to noise analysis. Noise characteristics between DLIR and ASiR-V, and their three selected levels were compared in terms of NPS peak frequency shift (shifts towards lower or higher spatial frequencies indicating coarser/grainier or finer noise textures, respectively), and noise magnitude ratio calculated according to the following formula:

Noise magnitude ratio $_{\mathrm{i}, \mathrm{j}}=\sqrt{\frac{\int \mathrm{NPS}\left(\text { dose }_{\mathrm{i}}, \text { algorithm }_{\mathrm{j}}\right)}{\int \mathrm{NPS}\left(\text { dose }_{\text {regular }}, \text { algorithm }_{\mathrm{ASiR}-\mathrm{V} 0 \%}\right)}}$

where $\mathrm{i}$ corresponds to regular, low or ultra-low, and $\mathrm{j}$ is ASiR-V $50 \%$ or $100 \%$ or DLIR low, medium or high. Noise magnitude ratios $\approx 0$ or 1 indicate substantial or no noise reductions, respectively.

\section{Target transfer function (TTF)}

Contrast-dependent spatial resolution was investigated in the two phantom regions with the appropriate object-to-background contrast inserts: low $(\mathrm{PE},|\Delta \mathrm{HU}| \approx 100 \mathrm{HU}$ ) and high (PTFE, $|\Delta \mathrm{HU}| \approx 1000 \mathrm{HU}$ ) contrast. Spatial resolution was quantified and characterized by computing TTFs as described in detail by Monnin et al. [35]. A total of 160 axial CT sections were used for TTF computations. Square ROIs of $272 \times 272$ pixels were semi-automatedly extracted from the CT image center to obtain 2D TTFs from edge spread functions, using angular aperture and pitch of $15^{\circ}$ and $10^{\circ}$, respectively. 1D TTFs were subsequently generated by radially averaging 2D TTFs. Spatial resolution performances between DLIR and ASiR-V, and their three selected levels were compared in terms of TTF at $50 \%\left(\mathrm{TTF}_{50}\right)$ and $10 \%\left(\mathrm{TTF}_{10}\right)$ of its maximum value.

\section{Nonprewhitening with eye filter (NPWE) model observer}

To consider and weight the combined effects of contrast, noise, and contrast-dependent spatial resolution on CT image quality, we computed detectability indexes (d') of simulated acute appendicitis or colonic diverticulitis (5-10-mm-diameter lesions with $|\Delta \mathrm{HU}| \approx 50 \mathrm{HU}$ ) [36] and calcium-containing urinary stones (2-6-mm-diameter lesions with $|\Delta \mathrm{HU}| \approx 500 \mathrm{HU}$ ) [37] using the nonprewhitening with eye filter (NPWE) model observer. This mathematical model observer was originally developed by Burgess [38] and later updated by Ott et al. [26] and Solomon et al. [39] to take into account the nonlinearity of IR techniques. The NPWE model observer mimics the diagnostic performance of the human eye for a detection task, with d' calculated according to the following formula:

$$
\mathrm{d}^{\prime}=\frac{\sqrt{2 \pi}|\Delta \mathrm{HU}| \int_{0}^{\mathrm{f}_{\mathrm{Ny}}} \mathrm{S}^{2}(\mathrm{f}) \operatorname{TTF}^{2}(\mathrm{f}) \operatorname{VTF}^{2}(\mathrm{f}) \mathrm{fdf}}{\sqrt{\int_{0}^{\mathrm{f}_{\mathrm{Ny}}} \mathrm{S}^{2}(\mathrm{f}) \mathrm{TTF}^{2}(\mathrm{f}) \mathrm{NPS}(\mathrm{f}) \mathrm{VTF}^{4}(\mathrm{f}) \mathrm{fdf}}}
$$

where $|\Delta \mathrm{HU}|$ is the absolute difference in CT numbers between the 

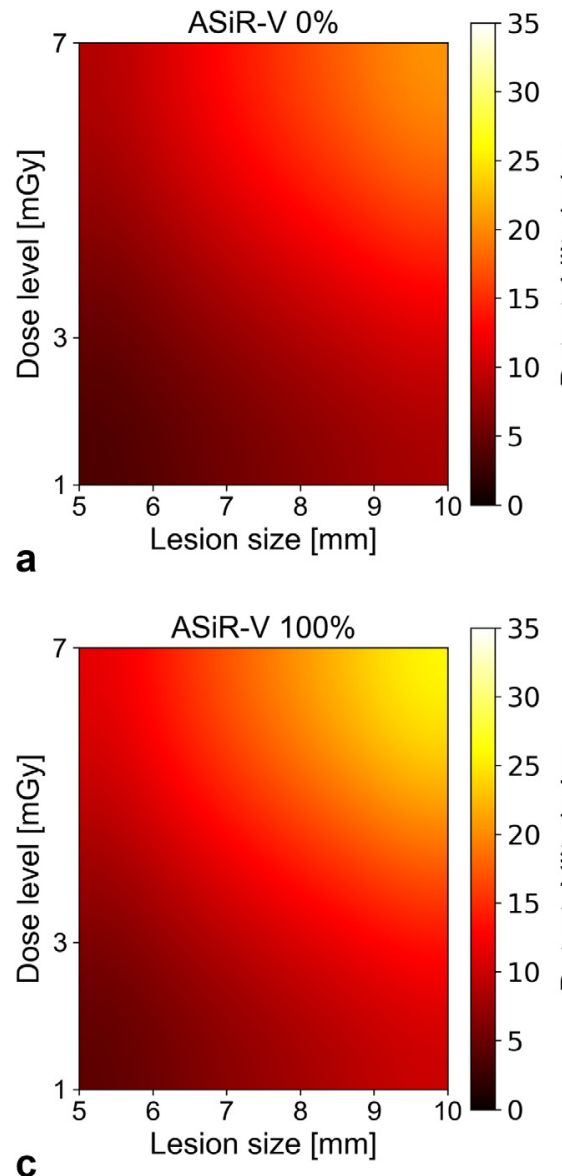

C

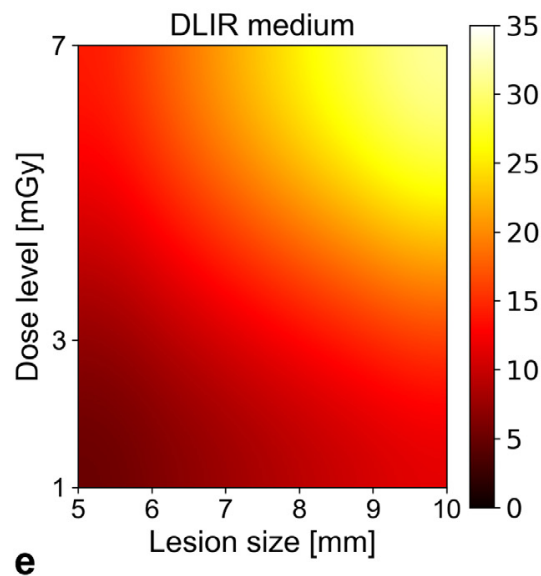

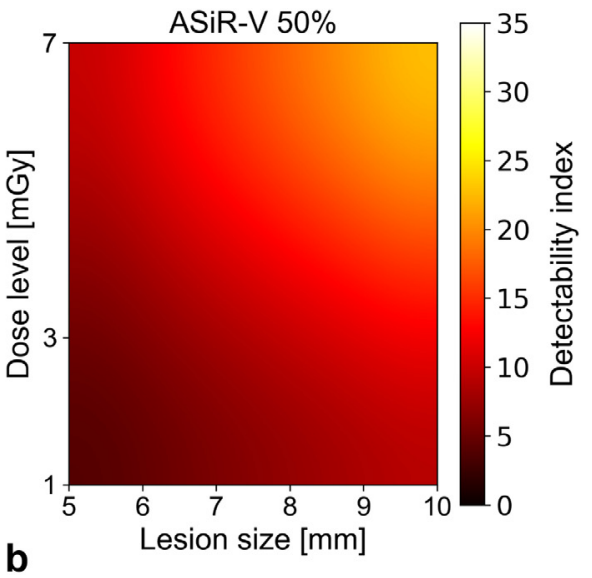
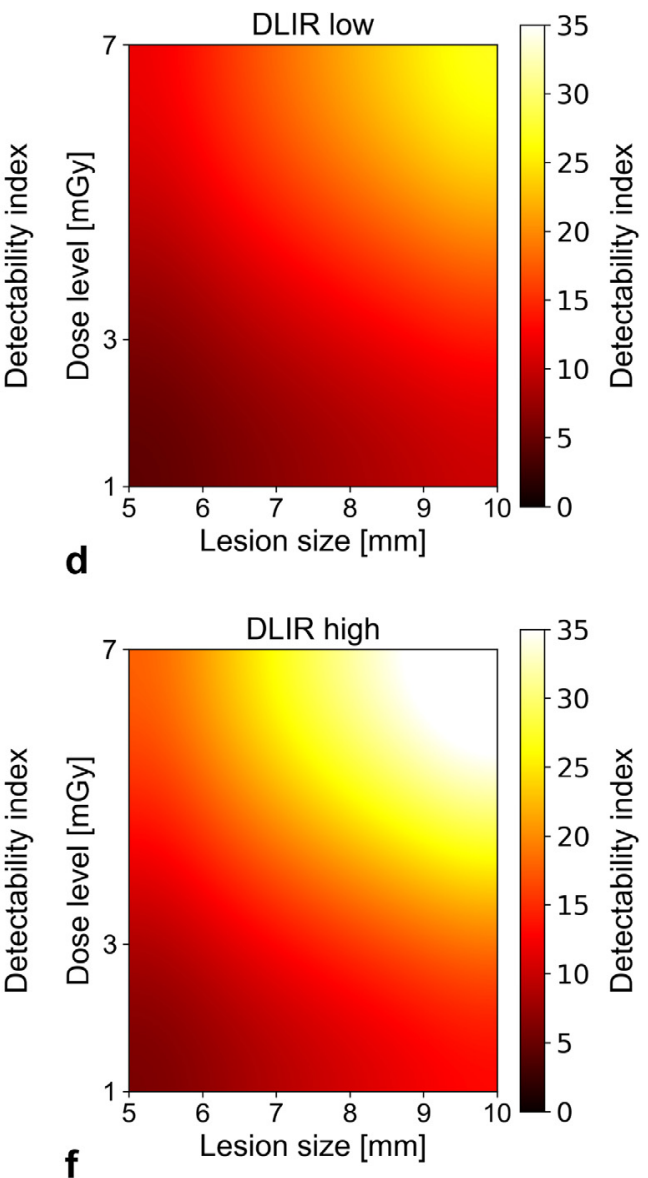

Fig. 6. Low-contrast detectability indexes (d') as a function of the image reconstruction technique (a, ASiR-V 0\%; b, ASiR-V 50\%; c, ASiR-V 100\%; d, DLIR low; e, DLIR medium; f, DLIR high), radiation dose level and simulated lesion size (suspected appendicitis or colonic diverticulitis). Overall, DLIR outperformed ASiR-V and increasing DLIR levels substantially enhanced detectability at any dose level for any simulated lesion size. $\mathrm{d}^{\prime} \geq 4.7$ corresponds to an AUC $\approx 1$. In this study, ASiR-V $0 \%$ was used as a surrogate for FBP.

object (target lesion) and homogenous background (water), $\mathrm{f}$ the radial spatial frequency, $\mathrm{f}_{\mathrm{Ny}}$ the radial Nyquist frequency, $\mathrm{S}$ the magnitude of the Fourier transform of the input signal (here, $S=\frac{r}{\mathrm{f}} J 1(2 \pi \mathrm{rf})$, with $\mathrm{r}$ the disk radius and $J_{1}$ the Bessel function of the first kind), and VTF the visual transfer function of the human eye [26]. Varying diameter disks represent different simulated lesions. d' can vary from 0 to infinity with d' $\geq 4.7$ corresponding to an area under the receiver operating characteristic curve $($ AUC) $\approx 1$.

In addition, we calculated potential dose savings with DLIR while maintaining comparable d' than with FBP or ASiR-V 50\%, for low- and high-contrast diagnostic tasks at regular (7 mGy) and low (3 mGy) doses, respectively.

\section{Results}

\subsection{Noise power spectrum}

The impact of DLIR on image noise as a function of dose level in comparison with FBP and ASiR-V is summarized in Figs. 2 and 3 and Tables 2 and 3. At all three investigated dose levels, increasing DLIR and ASiR-V levels both markedly decreased noise magnitude compared with FBP (Fig. 3). Overall, dose reductions shifted NPS towards lower 


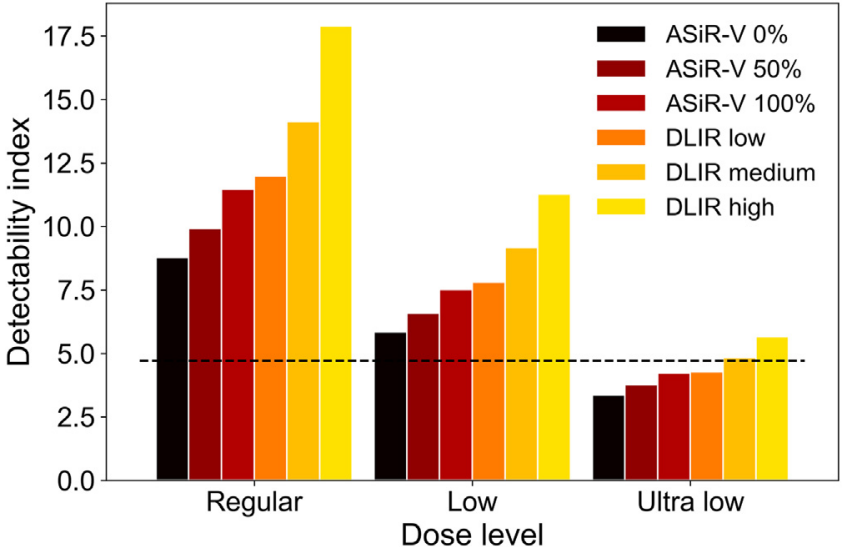

Fig. 7. Low-contrast detectability indexes (d') as a function of the radiation dose level for simulated 5-mm-diameter lesions with $|\Delta \mathrm{HU}| \approx 50 \mathrm{HU}$ (suspected appendicitis or colonic diverticulitis). Overall, DLIR outperformed ASiR$\mathrm{V}$ and increasing DLIR levels substantially enhanced detectability across all investigated dose levels. Only DLIR medium and high yielded $d^{\prime} \geq 4.7$ (dashed line, AUC $\approx 1$ ) at ultra-low dose. Using DLIR high at ultra-low dose, the potential dose savings while maintaining comparable d' than with FBP and ASiR-V $50 \%$ were $67 \%$ and $61 \%$, respectively.

spatial frequencies, with noise texture changes for both DLIR and ASiRV.

Relative noise magnitude ratios for DLIR and ASiR-V in comparison with regular-dose FBP at the three dose levels are reported in Table 2. At regular dose, relative noise magnitude ratios for DLIR high and ASiR$\mathrm{V} 100 \%$ were comparable, both at 0.4 (corresponding to $-84 \%$ difference with regular-dose FBP), while ratios for DLIR low and ASiR-V $50 \%$ were also comparable, both at $\sim 0.7$ (corresponding to $-54 \%$ difference with regular-dose FBP). Similar findings and trends were observed at low- and ultra-low doses, with comparable ratios for DLIR high and ASiR-V 100\% at ultra-low dose, both at $\sim 0.9$ (corresponding to -17 to $23 \%$ difference with regular-dose FBP).

NPS peak frequency shifts for DLIR and ASiR-V in comparison with FBP at the three dose levels are reported in Table 3. DLIR low and medium generally maintained noise texture compared with FBP at all dose levels, with slight NPS peak frequency shifts towards lower spatial frequencies, up to $\sim-10 \%$ for DLIR medium at regular dose. In contrast, DLIR high and ASiR-V changed noise texture compared with FBP regardless of dose, with moderate $~-15-20 \%$ shifts towards lower spatial frequencies. Noise texture alteration was more pronounced with increasing ASiR-V levels, with marked shifts of up to $\sim-50 \%$ for ASiR-V $100 \%$ at any dose level.

\section{Target transfer function}

The impact of DLIR on contrast-dependent spatial resolution as a function of dose level in comparison with FBP and ASiR-V is summarized in Figs. 4 and 5 and Tables 4 and 5. Overall, TTF decreased with decreasing dose levels for both DLIR and ASiR-V (Figs. 4 and 5).

For low-contrast $(|\Delta \mathrm{HU}| \approx 100 \mathrm{HU})$ spatial resolution, DLIR not only maintained, but even enhanced spatial resolution performances in comparison with FBP at all three investigated dose levels, particularly at low and ultra-low doses for $\mathrm{TTF}_{50}$, with up to $\sim 20 \%$ upgrades for DLIR low at low dose (Table 4). At regular dose, $\mathrm{TTF}_{50}$ slightly increased by $\sim 7-10 \%$ for any DLIR level, with no significant change in $\mathrm{TTF}_{10}$. Conversely, ASiR-V impaired spatial resolution compared with FBP regardless of dose (Table 4). Spatial resolution deterioration was more pronounced with increasing ASiR-V levels, exceeding $-30 \%$ downgrades for ASiR-V $100 \%$ at low and ultra-low doses.

For high-contrast $(|\Delta \mathrm{HU}| \approx 1000 \mathrm{HU})$ spatial resolution, DLIR generally maintained spatial resolution performances compared with
FBP at regular dose, while slightly to moderately enhancing both TTF metrics at low and ultra-low doses, with up to $\sim 20 \%$ upgrades in $\mathrm{TTF}_{50}$ (Table 5). In contrast, while ASiR-V 50\% did not significantly change spatial resolution regardless of dose, both $\mathrm{TTF}_{50}$ and $\mathrm{TTF}_{10}$ slightly decreased by $\sim-5-10 \%$ for ASiR-V $100 \%$ at low and ultra-low doses (Table 5).

\section{Nonprewhitening with eye filter model observer}

The impact of DLIR on d' as a function of dose and object-to-background contrast levels, and lesion size in comparison with FBP and ASiR-V are illustrated in Figs. 6-8. Overall, DLIR outperformed ASiR-V in all simulated clinical scenarios investigated.

For low-contrast diagnostic tasks (suspected appendicitis or colonic diverticulitis), increasing DLIR levels substantially enhanced d' at any dose and contrast levels for any simulated lesion size (Fig. 6). Only DLIR medium and high yielded $\mathrm{d}^{\prime} \geq 4.7$ (AUC $\approx 1$ ) for 5-mm-diameter lesions with $|\Delta \mathrm{HU}| \approx 50 \mathrm{HU}$ at ultra-low dose (Fig. 7).

Similar findings and trends were observed for high-contrast diagnostic tasks (suspected calcium-containing urinary stones), with d' ranging from 7.0 to 12.0 for FBP and DLIR high for 2-mm-diameter lesions with $|\Delta \mathrm{HU}| \approx 500 \mathrm{HU}$ at ultra-low dose, respectively (Fig. 8).

Using DLIR high, the potential dose savings while maintaining comparable $\mathrm{d}^{\prime}$ than with FBP and ASiR-V $50 \%$ for 5-mm-diameter lesions with $|\Delta \mathrm{HU}| \approx 50 \mathrm{HU}$ at ultra-low dose were $67 \%$ and $61 \%$, respectively (Fig. 7). In contrast, for the same simulated diagnostic task, the potential dose saving for ASiR-V 50\% compared with FBP was only $16 \%$. Similar dose savings estimates were found for 2-mm-diameter lesions with $|\Delta \mathrm{HU}| \approx 500 \mathrm{HU}$ at ultra-low dose.

\section{Discussion}

We thoroughly investigated a novel deep learning image reconstruction algorithm using state-of-the-art image quality metrics that account for the combined effects of noise, contrast and spatial resolution characteristics, in order to assess the impact of DLIR (TrueFidelity) on the detectability of clinically relevant, simulated, high- and lowcontrast abdominal lesions. In other words, this methodology translates comprehensive and robust CT system - including image reconstruction technique - performance evaluation into the ability to make an accurate clinical diagnosis through a detection task [40].

Our results indicate that DLIR decreases noise magnitude to a similar extent than ASiR-V, but can enhance spatial resolution and keep noise texture changes to a minimum in comparison with FBP. This translates into markedly improved lesion detectability indexes compared with FBP and ASiR-V, especially when using DLIR at its highest level, even for low-contrast diagnostic tasks and at ultra-low dose levels. The dose reduction potential provided by DLIR is therefore much higher than what statistical "hybrid" IR techniques can achieve. As with IR algorithms [19], the spatial resolution properties of DLIR depended on the object-to-background contrast level: for lower-contrast lesions, the TTF was lower than for high-contrast objects. Reducing the dose per se led to noise texture changes with all reconstruction algorithms; but at a given dose level, DLIR generally maintained noise texture, resulting in a preserved image appearance desirable for clinical radiologists.

As mentioned earlier, abdominal CT often implies low-contrast diagnostic tasks, sometimes with small focal lesions [1]. Many studies still only use simple contrast-to-noise ratios (CNR) as a surrogate metric for low-contrast detectability $[14,41]$. However, this first-order statistical metric could provide misleading results because it does not take into account lesion size and contrast (i.e. the method is not task-specific), nor noise texture $[42,43]$. Since low-contrast detectability is strongly affected by noise magnitude but also noise texture, fully characterizing the noise properties by decomposing its magnitude as a function of the spatial frequency content is a critical step in CT image quality assessment, especially when using IR techniques [40,43-46]. Detectability 

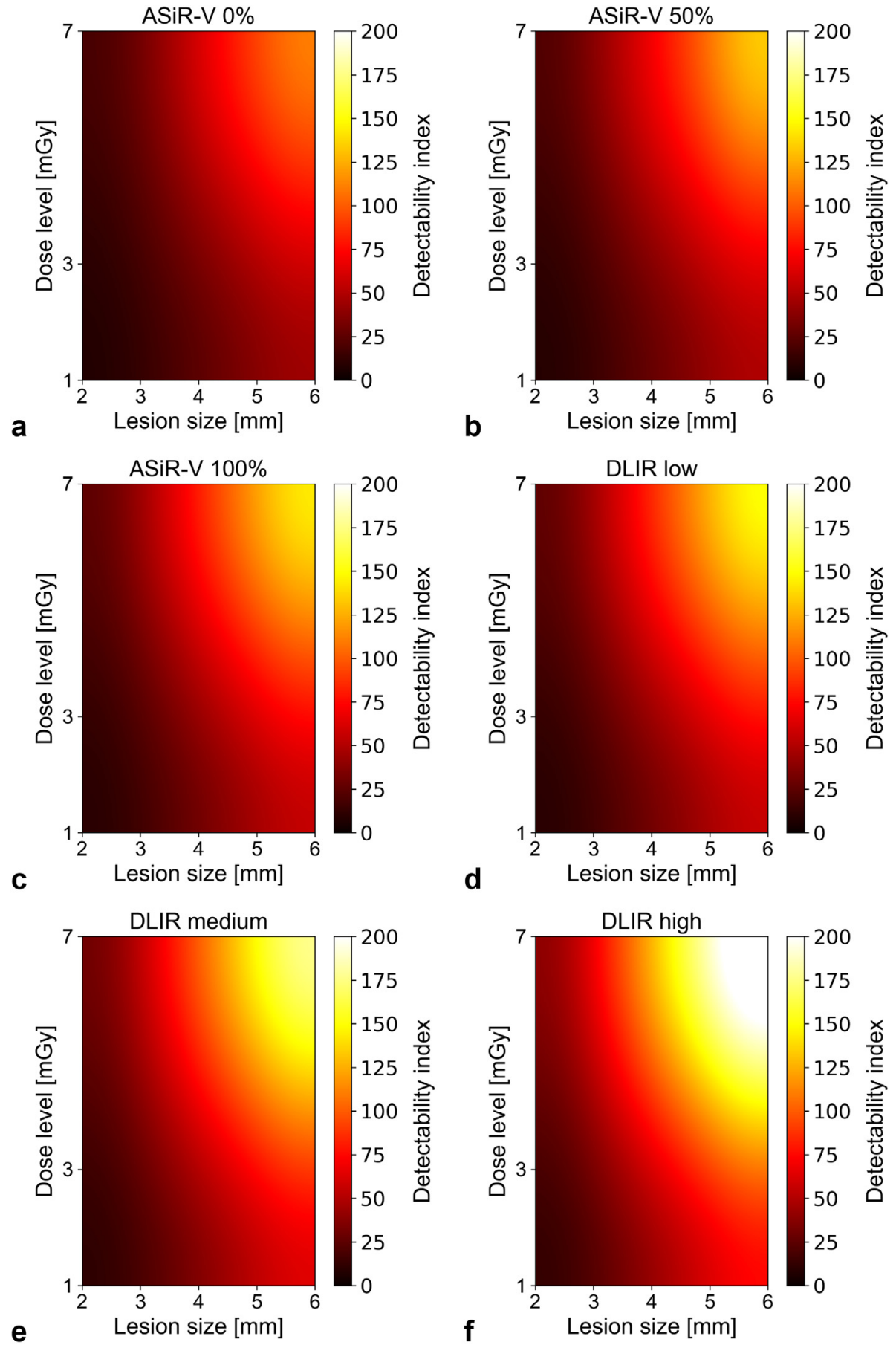

Fig. 8. High-contrast detectability indexes (d') as a function of the image reconstruction technique (a, ASiR-V 0\%; b, ASiR-V 50\%; c, ASiR-V 100\%; d, DLIR low; e, DLIR medium; f, DLIR high), radiation dose level and simulated lesion size (suspected calcium-containing urinary stones). Overall, DLIR outperformed ASiR-V and increasing DLIR levels substantially enhanced detectability at any dose level for any simulated lesion size. $\mathrm{d}^{\prime} \geq 4.7$ corresponds to an AUC $\approx 1$. In this study, ASiR-V $0 \%$ was used as a surrogate for FBP.

also strongly depends on signal magnitude and contrast, system resolution, and human visual system and viewing conditions. All the previous image quality characteristics can be integrated into mathematical model observers, such as the NPWE, to compute an overall detectability index. These model observers demonstrated a stronger correlation with human observer performance compared with CNR $[42,47]$. Therefore, DLIR promises a substantial impact on abdominal CT protocol optimization owing to its ability to decrease noise while maintaining its texture and improving spatial resolution, all at the same time.
The potential of DLIR in CT has recently been outlined in other clinical studies and scenarios. In a prospective study on 59 patients referred for standard-dose chest or abdominopelvic CT, Singh et al. performed an additional low-dose CT acquisition to evaluate the image quality and diagnostic performance of another DLIR algorithm: Advanced Intelligent Clear-IQ Engine (AiCE ${ }^{\circledR}$, Canon Medical) [48]. They concluded that submillisievert chest and abdominopelvic CT reconstructed with AiCE provides acceptable image quality, and all of the 39 pulmonary nodules detected at standard dose were also detected at low dose with DLIR, unlike with statistical and model-based IR. Noise 
and spatial resolution properties of CT images reconstructed with AiCE have been studied in a phantom study as well, in comparison with model-based IR (forward projected model-based iterative reconstruction, FIRST $^{\circledast}$, Canon Medical) [49]. Using dose levels ranging from 1.2 to $18.7 \mathrm{mGy}$, Higaki et al. computed NPS and TTF at different contrast levels. While model-based IR provided better spatial resolution at higher dose, DLIR images had lower noise and higher task-based detectability. In a further recent study on 43 patients undergoing coronary CT angiography reconstructed with DLIR (TrueFidelity) and ASiR-V, Benz et al. found that DLIR significantly reduced noise compared with ASiR-V while providing superior qualitative image quality and equal diagnostic accuracy [50].

Despite a careful design, our study has several limitations. First, CT image quality and, more specifically, lesion detectability was evaluated using a homogeneous phantom. To confirm superior lesion detectability with DLIR images, image quality assessment using a phantom with an anatomical background is needed. Second, we only investigated the impact of a vendor-specific DLIR algorithm (TrueFidelity) in comparison with a single partial model-based IR technique (ASiR-V), but a full model-based IR algorithm was not investigated because it was not available on the same CT system. Third, other DLIR methods from other CT manufacturers exist (e.g. AiCE, Canon Medical), yet we only assessed one of them (i.e. TrueFidelity, GE Healthcare) in this initial investigation. Fourth, we focused on a single reconstruction kernel only. Since several kernels are available on different CT systems and each kernel has its own noise texture, the assessment of additional kernels would be of interest for broader generalizability of our findings. Fifth, the detectability index $\left(d^{\prime}\right)$ models noise characteristics and contrastdependent spatial resolution properties to compute the diagnostic performance of a specific CT protocol and simulated clinical task, which means that our results can probably not be applied to any CT protocol for any clinical detection task. Finally, we did not directly compare the performance of human and mathematical model observers, but the model observer we used (NPWE) has been thoroughly validated against human observer performance $[42,47]$.

\section{Conclusions}

Increasing partial model-based IR (ASiR-V) level not only decreases image noise but also changes noise texture and impairs low-contrast detectability, particularly at reduced dose levels. Unlike ASiR-V, DLIR (TrueFidelity) substantially reduces noise while maintaining noise texture and slightly enhancing spatial resolution overall. DLIR outperforms ASiR-V for various simulated CT-based diagnostic tasks by enabling higher detectability of both low- and high-contrast focal abdominal lesions across all investigated dose levels. For low-contrast diagnostic tasks in particular, DLIR has the potential to enable substantial dose savings - far exceeding those achievable with current IR techniques - while maintaining lesion detectability and intrinsic diagnostic performance of abdominal CT examinations. The upcoming implementation of DLIR algorithms in routine clinical practice may mark the beginning of a new era for CT image reconstruction, clinical CT protocols and eventually CT diagnostic reference levels.

\section{Declaration of Competing Interest}

The authors declare that they have no known competing financial interests or personal relationships that could have appeared to influence the work reported in this paper.

\section{Acknowledgements}

David C. Rotzinger is supported by a grant from the Leenaards foundation. The authors are grateful to Martine Bernasconi and her team for their help with implementation of CT protocol settings and data acquisition.

\section{Funding source}

This research did not receive any specific grant from funding agencies in the public, commercial or not-for-profit sectors.

\section{Competing interests}

One of the authors (Brian Thomsen) is an employee of GE Healthcare. He had no control over data acquisition, analysis or interpretation.

\section{References}

[1] Stoker J, van Randen A, Laméris W, Boermeester MA. Imaging patients with acute abdominal pain. Radiology 2009;253:31-46. https://doi.org/10.1148/radiol. 2531090302.

[2] Rehani MM, Yang K, Melick ER, Heil J, Šalát D, Sensakovic WF, et al. Patients undergoing recurrent CT scans: assessing the magnitude. Eur Radiol 2020;30:1828-36. https://doi.org/10.1007/s00330-019-06523-y.

[3] Tonolini M, Valconi E, Vanzulli A, Bianco R. Radiation overexposure from repeated CT scans in young adults with acute abdominal pain. Emerg Radiol 2018;25:21-7. https://doi.org/10.1007/s10140-017-1554-y.

[4] White paper on radiation protection by the European Society of Radiology. Insights Imaging. 2011;2:357-62 DOI:10.1007/s13244-011-0108-1.

[5] Geyer LL, Schoepf UJ, Meinel FG, Nance Jr. JW, Bastarrika G, Leipsic JA, et al. State of the Art: iterative CT Reconstruction Techniques. Radiology 2015;276:339-57. https://doi.org/10.1148/radiol.2015132766.

[6] Willemink MJ, Leiner T, de Jong PA, de Heer LM, Nievelstein RA, Schilham AM, et al. Iterative reconstruction techniques for computed tomography part 2: initial results in dose reduction and image quality. Eur Radiol 2013;23:1632-42. https:// doi.org/10.1007/s00330-012-2764-z.

[7] Becce F, Ben Salah Y, Verdun FR, Vande Berg BC, Lecouvet FE, Meuli R, et al. Computed tomography of the cervical spine: comparison of image quality between a standard-dose and a low-dose protocol using filtered back-projection and iterative reconstruction. Skeletal Radiol 2013;42:937-45. https://doi.org/10.1007/s00256013-1576-9.

[8] den Harder AM, Willemink MJ, de Ruiter QM, Schilham AM, Krestin GP, Leiner T, et al. Achievable dose reduction using iterative reconstruction for chest computed tomography: a systematic review. Eur J Radiol 2015;84:2307-13. https://doi.org/ 10.1016/j.ejrad.2015.07.011.

[9] den Harder AM, Willemink MJ, van Doormaal PJ, Wessels FJ, Lock M, Schilham AMR, et al. Radiation dose reduction for CT assessment of urolithiasis using iterative reconstruction: a prospective intra-individual study. Eur Radiol 2018;28:143-50. https://doi.org/10.1007/s00330-017-4929-2.

[10] Greffier J, Pereira F, Macri F, Beregi JP, Larbi A. CT dose reduction using automatic exposure control and iterative reconstruction: a chest paediatric phantoms study. Phys Med 2016;32:582-9. https://doi.org/10.1016/j.ejmp.2016.03.007.

[11] Omoumi P, Verdun FR, Becce F. Optimization of radiation dose and image quality in musculoskeletal CT: emphasis on iterative reconstruction techniques (Part 2). Semin Musculoskelet Radiol 2015;19:422-30. https://doi.org/10.1055/s-00351569254.

[12] Tobalem F, Dugert E, Verdun FR, Dunet V, Ott JG, Rudiger HA, et al. MDCT arthrography of the hip: value of the adaptive statistical iterative reconstruction technique and potential for radiation dose reduction. AJR Am J Roentgenol 2014;203:W665-73. https://doi.org/10.2214/ajr.14.12821.

[13] Mileto A, Guimaraes LS, McCollough CH, Fletcher JG, Yu L. State of the art in abdominal CT: the limits of iterative reconstruction algorithms. Radiology 2019;293:491-503. https://doi.org/10.1148/radiol.2019191422.

[14] Schindera ST, Odedra D, Raza SA, Kim TK, Jang HJ, Szucs-Farkas Z, et al. Iterative reconstruction algorithm for CT: can radiation dose be decreased while low-contrast detectability is preserved? Radiology 2013;269:511-8. https://doi.org/10.1148/ radiol.13122349.

[15] Solomon J, Marin D, Roy Choudhury K, Patel B, Samei E. Effect of radiation dose reduction and reconstruction algorithm on image noise, contrast, resolution, and detectability of subtle hypoattenuating liver lesions at multidetector CT: filtered back projection versus a commercial model-based iterative reconstruction algorithm. Radiology 2017;284:777-87. https://doi.org/10.1148/radiol.2017161736.

[16] Viry A, Aberle C, Racine D, Knebel JF, Schindera ST, Schmidt S, et al. Effects of various generations of iterative CT reconstruction algorithms on low-contrast detectability as a function of the effective abdominal diameter: a quantitative taskbased phantom study. Phys Med 2018;48:111-8. https://doi.org/10.1016/j.ejmp. 2018.04.006.

[17] McCollough CH, Yu L, Kofler JM, Leng S, Zhang Y, Li Z, et al. Degradation of CT Low-Contrast Spatial Resolution Due to the Use of Iterative Reconstruction and Reduced Dose Levels. Radiology 2015;276:499-506. https://doi.org/10.1148/ radiol.15142047.

[18] Morsbach F, Desbiolles L, Raupach R, Leschka S, Schmidt B, Alkadhi H. Noise texture deviation: a measure for quantifying artifacts in computed tomography images with iterative reconstructions. Invest Radiol 2017;52:87-94. https://doi. 
org/10.1097/rli.0000000000000312.

[19] Rotzinger DC, Racine D, Beigelman-Aubry C, Alfudhili KM, Keller N, Monnin P, et al. Task-based model observer assessment of a partial model-based iterative reconstruction algorithm in thoracic oncologic multidetector CT. Sci Rep 2018;8:17734. https://doi.org/10.1038/s41598-018-36045-4.

[20] Laurent G, Villani N, Hossu G, Rauch A, Noël A, Blum A, et al. Full model-based iterative reconstruction (MBIR) in abdominal CT increases objective image quality, but decreases subjective acceptance. Eur Radiol 2019;29:4016-25. https://doi.org/ 10.1007/s00330-018-5988-8.

[21] Omoumi P, Verdun FR, Ben Salah Y, Vande Berg BC, Lecouvet FE, Malghem J, et al. Low-dose multidetector computed tomography of the cervical spine: optimization of iterative reconstruction strength levels. Acta Radiol 2014;55:335-44. https://doi. org $/ 10.1177 / 0284185113494981$.

[22] Jensen CT, Telesmanich ME, Wagner-Bartak NA, Liu X, Rong J, Szklaruk J, et al. Evaluation of abdominal computed tomography image quality using a new version of vendor-specific model-based iterative reconstruction. J Comput Assist Tomogr 2017;41:67-74. https://doi.org/10.1097/rct.0000000000000472.

[23] Miéville FA, Gudinchet F, Brunelle F, Bochud FO, Verdun FR. Iterative reconstruction methods in two different MDCT scanners: physical metrics and 4-alternative forced-choice detectability experiments-a phantom approach. Phys Med 2013;29:99-110. https://doi.org/10.1016/j.ejmp.2011.12.004.

[24] Willemink MJ, Noël PB. The evolution of image reconstruction for CT-from filtered back projection to artificial intelligence. Eur Radiol 2019;29:2185-95. https://doi. org/10.1007/s00330-018-5810-7.

[25] Ma XF, Fukuhara M, Takeda T. Neural network CT image reconstruction method for small amount of projection data. Nucl Instrum Methods Phys Res Sect A 2000;449:366-77. https://doi.org/10.1016/S0168-9002(99)01453-9.

[26] Ott JG, Becce F, Monnin P, Schmidt S, Bochud FO, Verdun FR. Update on the nonprewhitening model observer in computed tomography for the assessment of the adaptive statistical and model-based iterative reconstruction algorithms. Phys Med Biol 2014;59:4047-64. https://doi.org/10.1088/0031-9155/59/4/4047.

[27] Racine D, Viry A, Becce F, Schmidt S, Ba A, Bochud FO, et al. Objective comparison of high-contrast spatial resolution and low-contrast detectability for various clinical protocols on multiple CT scanners. Med Phys 2017;44:e153-63. https://doi.org/10. 1002/mp. 12224.

[28] Silva AC, Lawder HJ, Hara A, Kujak J, Pavlicek W. Innovations in CT dose reduction strategy: application of the adaptive statistical iterative reconstruction algorithm. AJR Am J Roentgenol 2010;194:191-9. https://doi.org/10.2214/ajr.09.2953.

[29] Thibault J-B, Sauer KD, Bouman CA, Hsieh J. A three-dimensional statistical approach to improved image quality for multislice helical CT. Med Phys 2007;34:4526-44. https://doi.org/10.1118/1.2789499.

[30] De Marco P, Origgi D. New adaptive statistical iterative reconstruction ASiR-V: Assessment of noise performance in comparison to ASiR. J Appl Clin Med Phys 2018;19:275-86. https://doi.org/10.1002/acm2.12253.

[31] Hsieh J, Liu E, Nett B, Tang J, Thibault J, Sahney S. A new era of imagereconstruction. TrueFidelity 2019.

[32] Boone JM, Brink JA, Edyvean S, Huda W, Leitz W, McCollough CH, et al. Radiation dose and image-quality assessment in computed tomography. J ICRU 2012;12:9-149. https://doi.org/10.1093/jicru/ndt006.

[33] P. Sharp D.C. Barber D.G. Brown A.E. Burgess C.E. Metz K.J. Myers et al. Report 54 Journal of the International Commission on Radiation Units and Measurements. NPNP 2016;os28: DOI:10.1093/jicru/os28.1.Report54.

[34] Miéville FA, Bolard G, Bulling S, Gudinchet F, Bochud FO, Verdun FR. Effects of computing parameters and measurement locations on the estimation of 3D NPS in non-stationary MDCT images. Phys Med 2013;29:684-94. https://doi.org/10. 1016/j.ejmp.2012.07.001.
[35] Monnin P, Bosmans H, Verdun FR, Marshall NW. A comprehensive model for quantum noise characterization in digital mammography. Phys Med Biol 2016;61:2083-108. https://doi.org/10.1088/0031-9155/61/5/2083.

[36] Frager D, Baer JW, Medwid SW, Rothpearl A, Bossart P. Detection of intestinal ischemia in patients with acute small-bowel obstruction due to adhesions or hernia: efficacy of CT. AJR Am J Roentgenol 1996;166:67-71. https://doi.org/10.2214/ajr. 166.1.8571907.

[37] Stewart G, Johnson L, Ganesh H, Davenport D, Smelser W, Crispen P, et al. Stone size limits the use of Hounsfield units for prediction of calcium oxalate stone composition. Urology 2015;85:292-5. https://doi.org/10.1016/j.urology.2014.10. 006.

[38] Burgess AE. Statistically defined backgrounds: performance of a modified nonprewhitening observer model. J Opt Soc Am A Opt Image Sci Vis 1994;11:1237-42. https://doi.org/10.1364/josaa.11.001237.

[39] Solomon J, Samei E. Correlation between human detection accuracy and observer model-based image quality metrics in computed tomography. J Med Imaging (Bellingham) 2016;3:035506https://doi.org/10.1117/1.Jmi.3.3.035506.

[40] Samei E, Bakalyar D, Boedeker KL, Brady S, Fan J, Leng S, et al. Performance evaluation of computed tomography systems: summary of AAPM task group 233. Med Phys 2019;46:e735-56. https://doi.org/10.1002/mp.13763.

[41] Baker ME, Dong F, Primak A, Obuchowski NA, Einstein D, Gandhi N, et al. Contrastto-noise ratio and low-contrast object resolution on full- and low-dose MDCT: SAFIRE versus filtered back projection in a low-contrast object phantom and in the liver. AJR Am J Roentgenol 2012;199:8-18. https://doi.org/10.2214/ajr.11.7421.

[42] Solomon J, Mileto A, Ramirez-Giraldo JC, Samei E. Diagnostic performance of an advanced modeled iterative reconstruction algorithm for low-contrast detectability with a third-generation dual-source multidetector CT Scanner: potential for radiation dose reduction in a multireader study. Radiology 2015;275:735-45. https:// doi.org/10.1148/radiol.15142005.

[43] Verdun FR, Racine D, Ott JG, Tapiovaara MJ, Toroi P, Bochud FO, et al. Image quality in CT: from physical measurements to model observers. Phys Med 2015;31:823-43. https://doi.org/10.1016/j.ejmp.2015.08.007.

[44] K.L. Boedeker M.F. McNitt-Gray Application of the noise power spectrum in modern diagnostic MDCT: part II. Noise power spectra and signal to noise Phys Med Biol. 52 2007 4047-61 DOI:10.1088/0031-9155/52/14/003.

[45] Burgess AE, Li X, Abbey CK. Visual signal detectability with two noise components: anomalous masking effects. J Opt Soc Am A Opt Image Sci Vis 1997;14:2420-42. https://doi.org/10.1364/josaa.14.002420.

[46] Siewerdsen JH, Cunningham IA, Jaffray DA. A framework for noise-power spectrum analysis of multidimensional images. Med Phys 2002;29:2655-71. https://doi.org/ $10.1118 / 1.1513158$.

[47] Racine D, Ba AH, Ott JG, Bochud FO, Verdun FR. Objective assessment of low contrast detectability in computed tomography with Channelized Hotelling Observer. Phys Med 2016;32:76-83. https://doi.org/10.1016/j.ejmp.2015.09.011.

[48] Singh R, Digumarthy SR, Muse VV, Kambadakone AR, Blake MA, Tabari A, et al. Image quality and lesion detection on deep learning reconstruction and iterative reconstruction of submillisievert chest and abdominal CT. AJR Am J Roentgenol 2020;214:566-73. https://doi.org/10.2214/ajr.19.21809.

[49] Higaki T, Nakamura Y, Zhou J, Yu Z, Nemoto T, Tatsugami F, et al. Deep learning reconstruction at CT: phantom study of the image characteristics. Acad Radiol 2020;27:82-7. https://doi.org/10.1016/j.acra.2019.09.008.

[50] Benz DC, Benetos G, Rampidis G, von Felten E, Bakula A, Sustar A, et al. Validation of deep-learning image reconstruction for coronary computed tomography angiography: impact on noise, image quality and diagnostic accuracy. J Cardiovasc Comput Tomogr 2020. https://doi.org/10.1016/j.jcct.2020.01.002. 